\title{
Bayesian Blind Source Separation of Positive Non Stationary Sources
}

\author{
Mahieddine M. ICHIR* and Ali MOHAMMAD-DJAFARI* \\ * Laboratoire des Signaux et Systèmes \\ Supélec, Plateau de Moulon, 91192 Gif sur Yvette, France
}

\begin{abstract}
In this contribution, we address the problem of blind non negative source separation. This problem finds its application in many fields of data analysis. We propose herein a novel approach based on Gamma mixture probability priors: Gamma densities to constraint the unobserved sources to lie on the positive half plane; a mixture density with a first order Markov model on the associated hidden variables to account for eventual non stationarity on the sources. Posterior mean estimates are obtained via appropriate Monte Carlo Markov Chain sampling.
\end{abstract}

\section{INTRODUCTION}

Blind source separation has been largely considered in the scientifi c community these last two decades, many solutions has been presented [1,9]. However in many data analysis applications (spectral analysis, image analysis ...) an additional constraint has to be enforced: positivity constraint on the sources and/or the mixing matrix elements. This is referred, in ICA community, as non negative Independent Component Analysis. In $[4,5]$, the authors considered a rectifi cation nonlinearity in non linear Principal Components to propose algorithms for this particular problem.

The authors in $[2,8,3]$ considered the non negative blind source separation under a Bayesian formulation: [2] considered a mixture of rectifi ed Gaussian prior to enforce such a constraint on both the sources and mixing elements. The author in [8] considered a same rectifi ed Gaussian prior to enforce such a constraint on the mixing elements. The authors in [3] considered a Gamma prior both for the sources and the mixing elements for a non negative source separation problem in mass spectrometry.

In this paper, we consider the problem of non negative source separation under a Bayesian framework, where we impose the positivity only on the sources (positivity constraint on the mixing elements can be accounted and the interested reader can refer to [3] for a possible extension). For that purpose, we model the sources by Gamma densities priors. We consider more particularly a non stationary prior distribution consisting of mixture of the Gamma densities, where the associated hidden variables are modeled by a fi rst order Markov chain.

Such prior models (Gamma densities) are highly non linear, leading to posterior joint densities highly intractable and making explicit analysis not possible. For the optimization purpose, we make use of Monte Carlo integration to estimate posterior means where we propose appropriate proposal distributions in Markov Chain Monte Carlo methods combining Gibbs and Hastings Metropolis sampling. 


\section{PROBLEM STATEMENT AND BAYESIAN FORMULATION}

The problem of instantaneous linear mixture blind source separation is described by:

$$
\boldsymbol{x}(t)=\boldsymbol{A s}(t)+\boldsymbol{\varepsilon}(t), \quad t \in \mathbb{Z}
$$

where $\boldsymbol{x}(t)$ and $\boldsymbol{\varepsilon}(t)$ are $m$ column vectors of the observed mixtures and observation noise respectively, $\boldsymbol{A}$ is the $(m \times n)$ unknown mixing matrix (containing the unknown mixing proportions of the sources) and $s(t)$ is a $n$ column vector of the unobservable sources. Each component of the unobservable sources $s(t)$ is constrained to lie on the positive half plane of $\mathbb{R}$. This is the case in many fi elds of data analysis as mass spectrometry for example. In the sequel, noise $\varepsilon(t)$ is assumed to be Gaussian spatially ${ }^{1}$ independent and temporarily white:

$$
\varepsilon(t) \sim \mathscr{N}\left(0, \boldsymbol{R}_{\varepsilon}\right), \quad t \in \mathbb{Z}
$$

We identify, in this problem, several unknown quantities to be estimated: the unobserved sources $\boldsymbol{s}(t)$, the mixing matrix $\boldsymbol{A}$, noise covariance $\boldsymbol{R}_{\varepsilon}$ and additional parameters that may describe the prior models (detailed hereafter). The joint posterior distribution, according to Bayes rule, is given by:

$$
p(s, \boldsymbol{A}, \boldsymbol{\theta} \mid \boldsymbol{x}) \propto p(\boldsymbol{x} \mid \boldsymbol{s}, \boldsymbol{A}, \boldsymbol{\theta}) \boldsymbol{\pi}(\boldsymbol{s}, \boldsymbol{A}, \boldsymbol{\theta})
$$

\section{Prior laws assignment}

We will make, first, assume that the sources $s(t)$ and the mixing matrix $\boldsymbol{A}$ are statistically a priori independent, i.e:

$$
\pi(s, A, \theta)=\pi(s \mid \theta) \pi(A \mid \theta)
$$

Mixing matrix prior distribution. The elements of the mixing matrix are considered a priori Gaussian and identically distributed:

$$
a_{i, j} \sim \mathscr{N}\left(\mu_{i, j}^{a}, \tau_{a}\right), \quad(i, j) \in\{1, \ldots, m\} \otimes\{1, \ldots, n\}
$$

This can be extended to the case where we the elements of the mixing matrix are constrained to lie on the positive half plane, however this is out of the scope of this paper and the reader can refer to [3] for further details.

Sources prior distribution. The sources constrained to lie on the positive half plane are modeled by Gamma prior distributions of the form:

$$
s_{i}(t) \sim \mathscr{G}\left(\alpha_{i}, \beta_{i}\right) \propto s_{i}(t)^{\alpha_{i}-1} e^{-s_{i}(t) / \beta_{i}} \mathbb{I}_{\mathbb{R}_{+}}, \quad t \in \mathbb{Z}
$$

\footnotetext{
${ }^{1}$ we refer to the vector components $s_{i}$ as the spatial dimension and to the time index $t$ as the temporal dimension
} 
with $\left\{\alpha_{i}, \beta_{i}\right\} \in\left\{\mathbb{R}_{+}^{*}, \mathbb{R}_{+}\right\}$. However, in order to account for any non stationarity of the sources, a mixture of these Gamma distributions is considered with a hidden variables modelisation of the form:

$$
\begin{aligned}
s_{i}(t) & \sim \sum_{k=1}^{K} p_{i, k} \mathscr{G}\left(s_{i}(t) \mid \alpha_{i, k}, \beta_{i, k}\right), \quad t \in \mathbb{Z} \\
& \sim \sum_{k=1}^{K} \operatorname{Prob}\left[z_{i}(t)=k\right] \mathscr{G}\left(s_{i}(t) \mid \alpha_{i, k}, \beta_{i, k}\right)
\end{aligned}
$$

with $\sum_{k=1}^{K} p_{i, k}=1$, where the sources, conditionally on the hidden variables, are Gamma densities of the form:

$$
\left(s_{i}(t) \mid z_{i}(t)=k\right) \sim \mathscr{G}\left(s_{i}(t) \mid \alpha_{i, k}, \beta_{i, k}\right)
$$

Hidden variables prior model: temporal non stationarity. In order to account for any non stationarity on the sources, the hidden variables are a prior modeled by a fi rst order Markov chain of the form:

$$
\pi\left[z_{i}(t)=k\right]=\sum_{k^{\prime}=1}^{K} \pi\left[z_{i}(t-1)=k^{\prime}\right] \pi\left[z_{i}(t)=k \mid z_{i}(t-1)=k^{\prime}\right]
$$

for $t=2, \ldots, T$ and $\pi\left[z_{i}(1)=k\right]=\pi_{1}(k)$.

Scale parameters prior distribution. The scale parameters are a priori modeled by inverse Gamma distributions of the form:

$$
\pi(\theta) \sim \mathscr{G}^{-1}\left(\theta \mid r_{0}, v_{0}\right) \propto \frac{1}{\theta^{r_{0}+1}} e^{-\frac{1}{v_{0} \theta}}
$$

where $\theta$ stands for the noise variances $\boldsymbol{R}_{\varepsilon}=\left[\sigma_{1}^{2}, \ldots, \sigma_{m}^{2}\right]^{\prime}$ and for the sources prior scale parameter $\beta_{i, k}$ as well.

Power parameter prior distribution. In order to defi ne an a priori distribution for the power parameter $\left\{\alpha_{i, k}\right\}$, we fi rst look at the likelihood of the parameter:

$$
\begin{aligned}
p\left(s_{i, 1 \ldots T} \mid z_{i, 1 \ldots T}=k, \alpha_{i, k}, \beta_{i, k}\right) & \propto \prod_{t_{k}=1}^{T_{i, k}} p\left(s_{i}\left(t_{k}\right) \mid z_{i}\left(t_{k}\right), \alpha_{i, k}, \beta_{i, k}\right) \\
& \propto\left(\frac{\left(\tilde{s}_{i, k} / \beta_{i, k}\right)^{\alpha_{i, k}}}{\Gamma\left(\alpha_{i, k}\right)}\right)^{T_{i, k}} \mathbb{I}_{\mathbb{R}_{+}}
\end{aligned}
$$

where $\tilde{s}_{i, k}=\sqrt[T_{i, k}]{s_{i}(1) \times \ldots \times s_{i}\left(T_{i, k}\right)}$. The geometrical mean $\tilde{s}_{i, k}$ presents a major drawback: it is null whenever one of the $s_{i}\left(t_{k}\right)$ 's is null, making thus the Gamma probability density function undefi ned at zero. In order to overcome this problem, the Gamma mixture prior of equation 7 needs to be redefi ned giving:

$$
s_{i}(t) \sim p_{i, 1} \mathscr{E x p}\left(s_{i}(t) \mid \lambda_{i}\right)+\sum_{k=2}^{K} p_{i, k} \mathscr{G}\left(s_{i}(t) \mid \alpha_{i, k}, \beta_{i, k}\right), \quad t \in \mathbb{Z}
$$


with $\left.\alpha_{i, k} \in\right] 1,+\infty\left[\right.$ and $\left\{\lambda_{i}, \beta_{i, k}\right\} \in \mathbb{R}_{+}$and a new constraint on the $\alpha_{i, k}$ 's.

\section{MONTE CARLO SAMPLING}

As an estimate of the different parameters of interest, we choose the posterior mean, and to achieve this we will approach it by means of Monte Carlo Markov Chain sampling. The posterior distribution 3 is quite intractable, thus Gibbs and Hastings-Metropolis procedures are alternated as described in the sequel:

Mixing matrix sampling. The posterior distribution of the mixing matrix conditioned on $(s, \theta)$ is given by:

$$
\begin{aligned}
p(\boldsymbol{A} \mid \boldsymbol{s}, \boldsymbol{\theta}, \boldsymbol{x}, \boldsymbol{\phi}) & \propto p\left(\boldsymbol{x} \mid \boldsymbol{s}, \boldsymbol{A}, \boldsymbol{R}_{\varepsilon}\right) \pi\left(\boldsymbol{A} \mid \mu_{A}, \boldsymbol{R}_{A}\right) \propto \mathscr{N}\left(\boldsymbol{x} \mid \boldsymbol{A} \boldsymbol{s}, \boldsymbol{R}_{\boldsymbol{\varepsilon}}\right) \mathscr{N}\left(\boldsymbol{A} \mid \mu_{A}, \boldsymbol{R}_{A}\right) \\
& \propto \mathscr{N}\left(\boldsymbol{A} \mid \hat{\mu}_{A}, \hat{\boldsymbol{R}}_{A}\right)
\end{aligned}
$$

where

$$
\begin{aligned}
\hat{\boldsymbol{R}}_{A} & =\left(\boldsymbol{R}_{\varepsilon}^{-1} \otimes \boldsymbol{R}_{s s}+\boldsymbol{R}_{A}^{-1}\right)^{-1} \\
\hat{\mu}_{A} & =\hat{\boldsymbol{R}}_{A}\left[\left(\boldsymbol{R}_{\varepsilon}^{-1} \otimes \mathbb{I}_{n}\right) \operatorname{Vect}\left(\boldsymbol{R}_{x s}\right)+\boldsymbol{R}_{A}^{-1} \mu_{A}\right]
\end{aligned}
$$

and $\boldsymbol{R}_{A}=\tau_{a} \mathbb{I}_{m n}, \mu_{A}=\left[\mu_{1,1}^{a}, \mu_{1,2}^{a}, \ldots, \mu_{m, n}^{a}\right]^{\prime}, \boldsymbol{R}_{s s}=\sum_{t} s s^{\prime}$ and $\boldsymbol{R}_{x s}=\sum_{t} \boldsymbol{x} \boldsymbol{s}^{\prime}$. Vect(.) is a row-wise column vectorization defi ned by:

$$
\operatorname{Vect}\left(\left[\begin{array}{ccc}
a_{1,1} & \ldots & a_{1, n} \\
\vdots & \ddots & \\
a_{m, 1} & \ldots & a_{m, n}
\end{array}\right]\right)=\left[a_{1,1}, a_{1,2}, \ldots, a_{m, n}\right]^{\prime}
$$

Noise variance sampling. The noise conditional posterior distribution is given by:

$$
\begin{aligned}
p\left(\tau_{i} \mid \boldsymbol{x}, \boldsymbol{A s}\right) & \propto p\left(\boldsymbol{x}_{i} \mid[\boldsymbol{A} \boldsymbol{s}]_{i}, \tau_{i}\right) \pi\left(\tau_{i}\right) \propto \mathscr{N}\left(\boldsymbol{x}_{i} \mid[\boldsymbol{A} \boldsymbol{s}]_{i}, \tau_{i}\right) \mathscr{G}^{-1}\left(\tau_{i} \mid r_{0}, v_{0}\right) \\
& \propto \mathscr{G}^{-1}\left(\tau_{i} \mid r, v\right)
\end{aligned}
$$

for $i=1, \ldots, m$ where $r=T / 2+r_{0}$ and

$$
\frac{1}{v}=\frac{1}{2} \sum_{t}\left(x_{i}(t)-[\boldsymbol{A s}(t)]_{i}\right)^{2}+\frac{1}{v_{0}}
$$

Gamma priors scale parameter sampling. The conditional posterior distribution of the scale parameter of the mixture prior (equation 7) is given by:

$$
\begin{aligned}
p\left(\beta_{i, k} \mid s_{i, 1 \ldots T_{k}}\right) & \propto p\left(s_{i, 1 \ldots T_{k}} \mid \beta_{i, k}\right) \pi\left(\beta_{i, k}\right) \propto \mathscr{G}\left(s_{i, 1 \ldots T_{k}} \mid \alpha_{i, k}, \beta_{i, k}\right) \mathscr{G}^{-1}\left(\beta_{i, k} \mid r_{0}, v_{0}\right) \\
& \propto \mathscr{G}^{-1}\left(\beta_{i, k} \mid r, v\right)
\end{aligned}
$$


for $i=1, \ldots, n ; k=1, \ldots, K$ where $r=\alpha_{i, k} T_{i, k} / 2+r_{0}$ and $^{2}$

$$
\frac{1}{v}=\sum_{t_{k}=1}^{T_{i, k}} s_{i}\left(t_{k}\right)+\frac{1}{v_{0}}
$$

Power parameter sampling. The conditional posterior probability density function of the power parameter of the Gamma mixture prior (equation 7) is given by:

$$
\begin{aligned}
p\left(\alpha_{i, k} \mid s_{i, 1 \ldots T_{k}}, \beta_{k}\right) & \propto p\left(s_{i, 1 \ldots T_{k}} \mid \beta_{i, k}, \alpha_{i, k}\right) \pi\left(\alpha_{i, k}\right) \propto\left(\frac{\left(\tilde{s}_{i, k} / \beta_{i, k}\right)^{\alpha_{i, k}}}{\Gamma\left(\alpha_{i, k}\right)}\right)^{T_{i, k}} \mathbb{I}_{] 1,+\infty[} \\
& \propto\left(\frac{w^{\alpha_{i, k}}}{\Gamma\left(\alpha_{i, k}\right)}\right)^{T_{i, k}} \mathbb{I}_{] 1,+\infty[}
\end{aligned}
$$

$w=\tilde{s}_{i, k} / \beta_{i, k}$. The distribution between brackets would be the Poisson distribution for $\alpha_{i, k}$ if the latter would have been discrete.

We make use of the Metropolis Hastings algorithm in order to generate conditional posterior samples from distribution 16 with a proposal distribution given by:

$$
g(y)=\mathscr{G}(y \mid \vartheta, \gamma)
$$

$\vartheta$ and $\gamma$ are such that:

$$
\left\{\begin{array}{l}
\vartheta=\hat{\alpha}^{2} / \varphi \\
\gamma=\varphi / \hat{\alpha}
\end{array}\right.
$$

where $\Psi(\alpha)=\frac{\partial \ln \Gamma}{\partial \alpha}(\alpha), \hat{\alpha}=\Psi^{-1}(\ln (w))$ and $\varphi=\frac{1}{T_{i, k}}\left(\frac{\partial \Psi}{\partial \alpha}(\vartheta)\right)^{-1}$.

Sampling the hidden variables. Two alternatives are possible to sample the hidden variables with a Markov chain prior model (equation 9): an exact method based on the Baum \& Walsh procedure [6] and an approximate one based on Gibbs sampling procedure [7]. In this work, we adopt the second one being simpler and faster to implement, it is detail hereafter:

$$
\begin{aligned}
\left(z_{i}(t)=k \mid s_{i}(t), z_{i,-t}, \theta_{k}\right) \sim & \mathscr{G}\left(s_{i}(t) \mid \alpha_{i, k}, \beta_{i, k}\right) \\
& \times \pi\left[z_{i}(t+1) \mid z_{i}(t)=k\right] \pi\left[z_{i}(t)=k \mid z_{i}(t-1)\right]
\end{aligned}
$$

for $t=2, \ldots, T-1$, where $z_{i,-t}=\left[\ldots, z_{i}(t-1), z_{i}(t+1), \ldots\right]$ and

$$
\begin{aligned}
\left(z_{i}(1)=k \mid s_{i}(1), z_{i,-t}, \theta_{k}\right) & \sim \mathscr{G}\left(s_{i}(1) \mid \alpha_{i, k}, \beta_{i, k}\right) \pi\left[z_{i}(2) \mid z_{i}(1)=k\right] \\
\left(z_{i}(T)=k \mid s_{i}(T), z_{i,-t}, \theta_{k}\right) & \sim \mathscr{G}\left(s_{i}(T) \mid \alpha_{i, k}, \beta_{i, k}\right) \pi\left[z_{i}(T) \mid z_{i}(T-1)=k\right]
\end{aligned}
$$

2 recall that $\beta_{i, 1}=\lambda_{i}$ and $\alpha_{i, 1}=1$ 
Sampling the sources. The conditional posterior distribution of the sources is given by:

$$
\begin{aligned}
p(\boldsymbol{s} \mid \boldsymbol{x}, \boldsymbol{z}, \boldsymbol{A}, \Theta) & \propto p\left(\boldsymbol{x} \mid \boldsymbol{s}, \boldsymbol{A}, \boldsymbol{R}_{\varepsilon}\right) \pi(\boldsymbol{s} \mid \boldsymbol{z}, \boldsymbol{\theta}) \\
& \propto\left(\prod_{i=1}^{n} s_{i}^{\alpha_{i, k}-1}\right) \exp \left(-\frac{1}{2} \boldsymbol{Q}(\boldsymbol{s} \mid \boldsymbol{x}, \boldsymbol{A}, \Theta)\right)
\end{aligned}
$$

where $\boldsymbol{Q}($.$) is a quadratic function on s$ given by:

$$
\boldsymbol{Q}(s \mid \boldsymbol{x}, \boldsymbol{A}, \Theta)=\left(s-\mu_{s}\right)^{\prime} \Sigma\left(s-\mu_{s}\right)
$$

where $\Sigma=\boldsymbol{A}^{\prime} \boldsymbol{R}_{\varepsilon}^{-1} \boldsymbol{A}$ and $\mu_{s}=\Sigma^{-1}\left(\boldsymbol{A}^{\prime} \boldsymbol{R}_{\varepsilon}^{-1} \boldsymbol{x}-\boldsymbol{\beta}^{-1}\right)^{3}$. A hybrid Gibbs HastingsMetropolis scheme is used to generate samples from the distribution 18:

The conditional posterior distribution of source $s_{i}$ is given by:

$$
p\left(s_{i}\right) \propto s_{i}^{\alpha_{i, k}-1} e^{-\frac{\zeta_{i}}{2}\left(s_{i}-\mu_{i}\right)^{2}} \mathbb{I}_{\mathbb{R}_{+}}
$$

where $\zeta_{i}=\Sigma[i, i]$ and $\mu_{i}=\left(\left[\Sigma \mu_{s}\right]_{i}-\sum_{j \neq i}^{n} \Sigma[i, j] s_{j}\right) / \Sigma[i, i]$. We distinguish mainly three cases:

$\underline{\mu_{i}=0}:$ the distribution 19 rewrites

$$
p\left(s_{i}\right) \propto s_{i}^{\alpha_{i}-1} e^{-\frac{\zeta_{i}}{2} s_{i}^{2}} \mathbb{I}_{\mathbb{R}_{+}}
$$

In this case $s_{i}=\sqrt{y / \zeta_{i}}$ where $y \sim \chi^{2}\left(\alpha_{i, k}\right) \propto y^{\alpha_{i, k} / 2-1} e^{-y / 2} \mathbb{I}_{\mathbb{R}_{+}}$. $\mu_{i}<0$ : we can rewrite the distribution 19 as:

$$
p\left(s_{i}\right) \propto s_{i}^{\alpha_{i, k}-1} e^{-\zeta_{i}\left|\mu_{i}\right| s_{i}} e^{-\frac{\zeta_{i}}{2} s_{i}^{2}} \mathbb{I}_{\mathbb{R}_{+}}
$$

then $y \sim \mathscr{G}\left(y \mid \alpha_{i, k}, \frac{1}{\zeta_{i}\left|\mu_{i}\right|}\right)$, where the rejection probability is given by:

$$
\rho=\min \left(1, e^{-\frac{\zeta_{i}}{2}\left(y^{2}-s_{i}^{(l), 2}\right)}\right)
$$

the quantity $l$ stands for the iteration index.

$\underline{\mu_{i}>0}$ : in this particular case, a proposal distribution is given by:

$$
g(y) \propto y^{\alpha_{i, k}-1} e^{-\zeta_{i}\left|y-\mu_{i}\right|} \mathbb{I}_{\mathbb{R}_{+}}
$$

with a rejection probability given by

$$
\rho^{\prime}=\left\{\begin{array}{lll}
e^{-\frac{\zeta_{i}}{2}\left(y-s_{i}^{l-1}\right)\left(y+s_{i}^{l-1}-2 \mu_{i}-2\right)} & \text { when } \quad\left(y-\mu_{i}, s_{i}^{l-1}-\mu_{i}\right) \geq 0, \\
e^{-\frac{\zeta_{i}}{2}\left(y-s_{i}^{l-1}+2\right)\left(y+s_{i}^{l-1}-2 \mu_{i}\right)} & \text { when } \quad\left(\mu_{i}-y, s_{i}^{l-1}-\mu_{i}\right) \geq 0 \\
e^{-\frac{\zeta_{i}}{2}\left(y-s_{i}^{l-1}-2\right)\left(y+s_{i}^{l-1}-2 \mu_{i}\right)} & \text { when } \quad\left(y-\mu_{i}, \mu_{i}-s_{i}^{l-1}\right) \geq 0 \\
e^{-\frac{\zeta_{i}}{2}\left(y-s_{i}^{l-1}\right)\left(y+s_{i}^{l-1}-2 \mu_{i}+2\right)} & \text { when } \quad\left(\mu_{i}-y, \mu_{i}-s_{i}^{l-1}\right) \geq 0
\end{array}\right.
$$

${ }^{3} \beta^{-T}$ defines the point wise inverse of the vector $\beta$ 
and $\rho=\min \left(1, \rho^{\prime}\right)$.

\section{SIMULATIONS}

The proposed approach has been tested on the data set presented on fi gure 1: the two sources (middle) are realizations of Gamma mixture densities with the associated discrete variables (top). The observations (bottom) have been obtained with a mixing ma$\operatorname{trix} \boldsymbol{A}=[[1, .5],[.5,1]]$ and a low level centered white Gaussian noise (the observation signal to noise ratio (SNR) is of about $25 \mathrm{~dB}$ ), with:

$$
\operatorname{SNR}_{\mathrm{dB}}(i)=10 \log \left(\frac{\left\|[\boldsymbol{A} * \boldsymbol{s}(t)]_{i}\right\|_{2}^{2}}{\left\|\varepsilon_{i}(t)\right\|_{2}^{2}}\right), \quad i=1, \ldots, m
$$
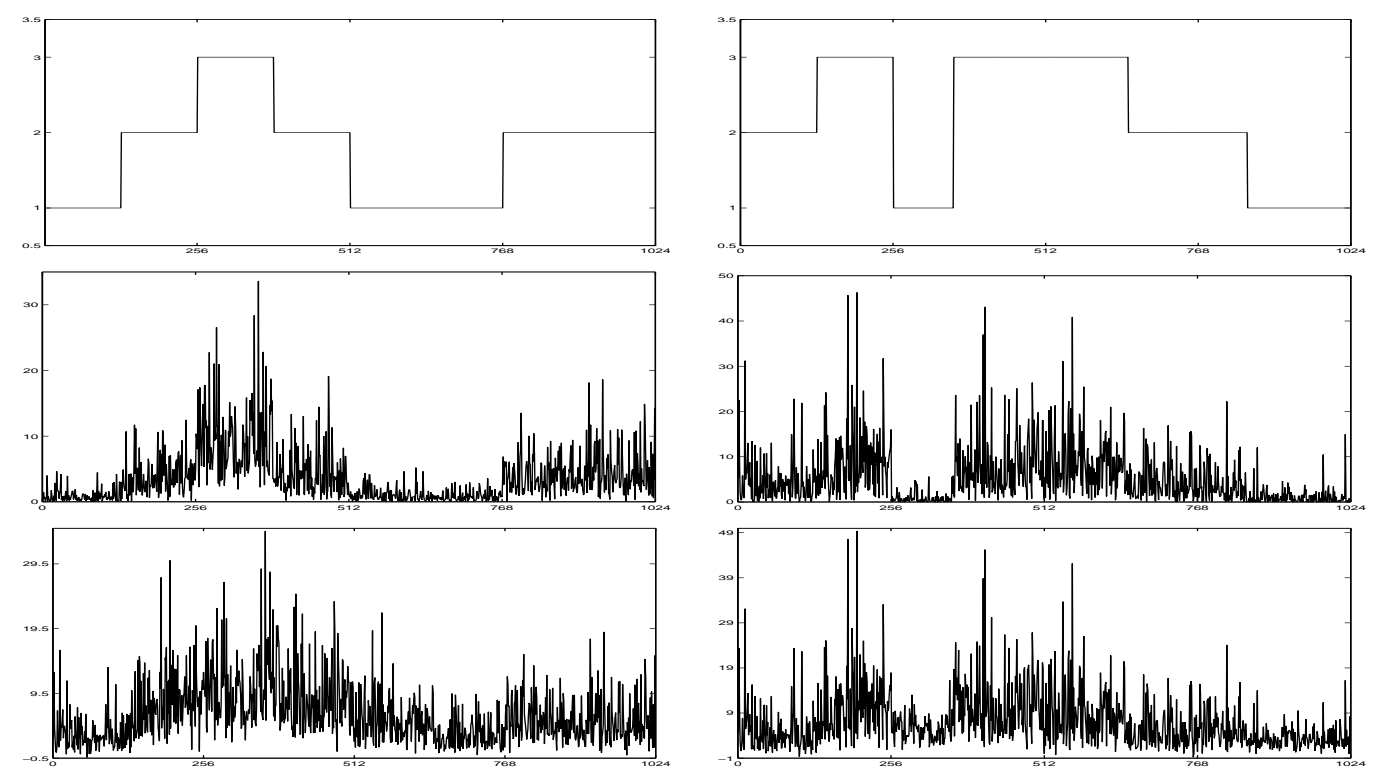

FIGURE 1. Hidden variables representing the different states of the original sources at different time instants (top); original unobserved sources (middle), observations (bottom)

On fi gure 2, we present the obtained estimates with the outlined MCMC algorithm: the estimated hidden states (top) and the estimated sources (bottom). We give, for comparison, the proportions of the miss-estimated hidden states for each source. For this let us fi rst defi ne:

$$
\eta_{i}(k)=\frac{\operatorname{Card}\left\{t \mid \hat{z}_{i}(t)=k\right\}-\operatorname{Card}\left\{t \mid z_{i}(t)=k\right\}}{\operatorname{Card}\left\{t \mid z_{i}(t)=k\right\}}
$$

then

$$
\begin{aligned}
& \eta_{1}=[.028646, .285156, .101562] \\
& \eta_{2}=[.036364, .246951, .370546]
\end{aligned}
$$



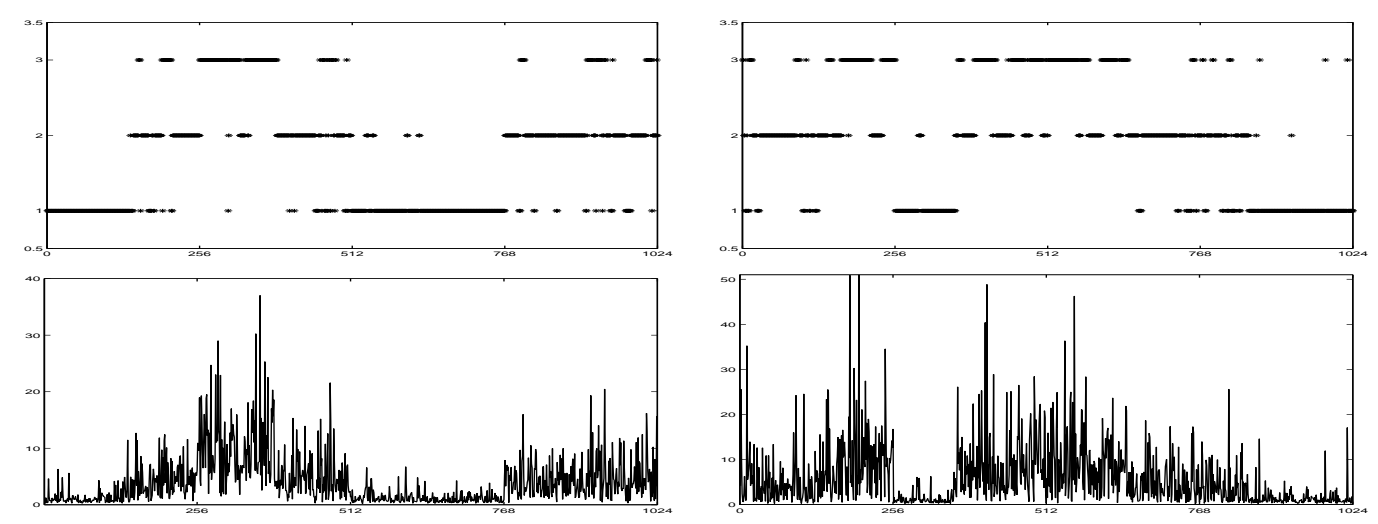

FIGURE 2. Estimated data: a. Hidden variables representing the different states of the sources at different time instants, b. Estimated unobserved sources

\section{CONCLUSION}

We presented in this paper a novel approach for separating non negative sources in a blind source separation problem based on a Bayesian formulation of the problem. The proposed approach is based on Markov Chain Monte Carlo methods. The prior model for the sources is a Gamma mixture model: the Gamma density function constrains the sources to lie on the right half plane while the mixture model enables to model any sources non stationarity. Simulation results allowed us to show the performances of the proposed approach.

A direct extension of this work would be to incorporate an estimation step for the number of the hidden states, and a natural way to do this in a Bayesian approach with MCMC methods is through Reversible Jump sampling.

\section{REFERENCES}

1. Aapo Hyvärinen, Juha Karhunen, and Erkki Oja. Independent Component Analysis. John Wiley, New York, 2001.

2. James W. Miskin. Ensemble Learning for Independant Component Analysis. PhD thesis, Selwyn College, Cambridge University, 2000.

3. Saïd Moussaoui, David Brie, Olivier Caspary, and Ali Mohammad-Djafari. A Bayesian method for positive source separation. In ICASSP04, October 2004.

4. Erkki Oja. Independant component analysis and blind source separation. Technical report, Helsinki University of Technology, 2003.

5. Erkki Oja and Mark Plumbley. Blind separation of positive sources using non-negative pca. In Independant Component Analysis and Blind Source Separation, Nara, Japan, April 2003.

6. Lawrence R. Rabiner. A tutorial on hidden Markov models and selected applications in speech recongnition. volume 77, pages 257-286, February 1989.

7. Christian P. Robert, Gilles Celeux, and Jean Diebolt. Bayesian estimation of hidden Markov models: a stochastic implementation. Statistics and Probability Letters, 16:77-83, 1993.

8. S.J. Roberts and Rizwan A. Choudrey. Data decomposition using independant component analysis with prior constraints. Pattern Recognition, 2003.

9. Hichem Snoussi. Bayesian approach to source separation. Applications in imagery. PhD thesis, University of Paris-Sud, Orsay, France, september 2003. 